\title{
2. Definición, patogenia y factores de riesgo de la fibrosis pulmonar idiopática
}

\author{
FELIPE REYES C.*
}

\section{Definition, pathogenesis and risk factors of idiopathic pulmonary fibrosis}

Idiopathic pulmonary fibrosis (IPF) is a specific form of idiopathic interstitial pneumonia, of chronic and progressive fibrosing type, with radiological and / or histological pattern of usual interstitial pneumonia (UIP). Its pathogenesis is complex, the most accepted model currently is based on the fact that the alveolar epithelial cells, aberrantly activated, lead to the proliferation of fibroblasts and their differentiation to myofibroblasts that deposit extracellular matrix and irreversibly destroy the pulmonary architecture. There is no clear initial trigger that explains the activation and subsequent maintenance of the fibrosis mechanism. The transforming growth factor beta (TGF- $\beta)$, released by the alveolar epithelial cells, has been implicated as one of the main drivers of the induction and proliferation of altered fibroblasts that persists long after the initial stimulation, which would largely explain progressive and chronic clinical behavior.

Key words: Idiopathic pulmonary fibrosis; Myofibroblasts; Alveolar Epithelial Cells, Extracellular Matrix; Transforming Growth Factor beta.

\section{Resumen}

La fibrosis pulmonar idiopática (FPI) es una forma específica de neumonía intersticial idiopática, de tipo fibrosante crónica y progresiva, con patrón radiológico y/o histológico de neumonía intersticial usual (NIU). Su patogenia es compleja, el modelo más aceptado actualmente es basado en las células epiteliales alveolares, aberrantemente activadas que conducen a la proliferación de fibroblastos y su diferenciación a miofibroblastos que depositan matriz extracelular y destruyen irreversiblemente la arquitectura pulmonar. No existe un claro factor inicial que explique la activación y posterior mantención del mecanismo de la fibrosis. El factor de crecimiento transformante beta (TGF- $\beta$ ) liberado por las células epiteliales alveolares se ha implicado como unos de los principales conductores de la inducción y proliferación de fibroblastos alterados que persiste mucho tiempo después de la estimulación inicial, lo que explicaría en gran parte el comportamiento clínico progresivo y crónico.

Palabras clave: Fibrosis pulmonar idiopática, Miofibroblastos; Células Epitaliales Alveolares, Matriz Extracelula; Factor de Crecimiento Transformante beta.

La fibrosis pulmonar idiopática (FPI) es una forma específica de neumonía intersticial idiopática, de tipo fibrosante crónica y progresiva, con patrón radiológico y/o histológico de neumonía intersticial usual (NIU) ${ }^{1}$. Su patogenia es compleja: inicialmente, basada en modelos animales, se presumió un origen inflamatorio que posteriormente daría paso al desarrollo de una respuesta fibrótica. Sin embargo, el modelo más aceptado ac- tualmente es basado en que las células epiteliales alveolares, aberrantemente activadas, conducen a la proliferación de fibroblastos y su diferenciación a miofibroblastos que depositan cantidades excesivas de matriz extracelular y destruyen irreversiblemente la arquitectura pulmonar. Si bien las microlesiones recurrentes en la pared alveolar y las vías aéreas distales parecen ser necesarias para la activación del epitelio y la transforma-

* Coordinador Comisión de Enfermedades Pulmonares Intersticiales Difusas, SER Chile. Hospital Clínico Universidad de Chile. Instructor Universidad de Chile. Santiago, Chile. 
ción de la transición epitelio-mesénquima, no se han identificado factores extrínsecos claramente causales. El tabaquismo, la infección viral, los contaminantes ambientales, la aspiración crónica y la predisposición genética, han sido implicados como posibles gatillantes contribuyentes.

La progresión es la característica distintiva de la FPI. Es posible que la lesión inicial del epitelio y la liberación de citoquinas provoquen efectos duraderos en los fibroblastos. En modelos experimentales, la liberación del factor de crecimiento transformante beta (TGF- $\beta$ ) por las células epiteliales alveolares conduce a la inducción y proliferación de fibroblastos alterados que persiste mucho tiempo después de la estimulación inicial $^{2}$ (Figura 1).

Algunos investigadores proponen eliminar el término "idiopática" de su definición, debido a avances sustanciales en los mecanismos genéti$\cos$, ambientales, celulares y moleculares involucrados en su desarrollo. Algunas de las definiciones alternativas propuestas son: fibrosis pulmonar (FP) activada por epitelio y clasificaciones nuevas basadas en criterios clínico-etiológicos (FP comandada por disfunción de células epiteliales;
FP comandada por disfunción de células inflamatorias; FP ocupacionales o inducidas por drogas (asbestosis, silicosis, medicamentos, FP debida a tabaquismo) $)^{4}$. Esta postura contrasta con otra vertiente, que propone agrupar las enfermedades fibrosantes progresivas (FPI, neumonitis por hipersensibilidad crónica, NIU secundaria a artritis reumatoide, etc), ya que comparten evoluciones, pronósticos $\mathrm{y}$, eventualmente, tratamientos similares $^{5,6}$.

La causa de la FPI es desconocida. Sin embargo, se han descrito exposiciones ambientales y ocupacionales que podrían estar asociadas a su patogénesis, como factores de riesgo: la FPI es más común en exfumadores o fumadores acti$\operatorname{vos}^{7}$, también se han descrito como factores de riesgo la exposición a corte o pulido de piedra, a polvo de metal y madera, acero, latón o plomo ${ }^{8}$; la inhalación química, los solventes orgánicos y la residencia en áreas agrícolas o urbanas contaminadas 9 .

Un meta-análisis de 6 estudios caso-control encontró 6 exposiciones asociadas a FPI: tabaquismo, actividades agrícolas, ganadería, polvo de madera, polvo de metal y de piedra/arena ${ }^{10}$.

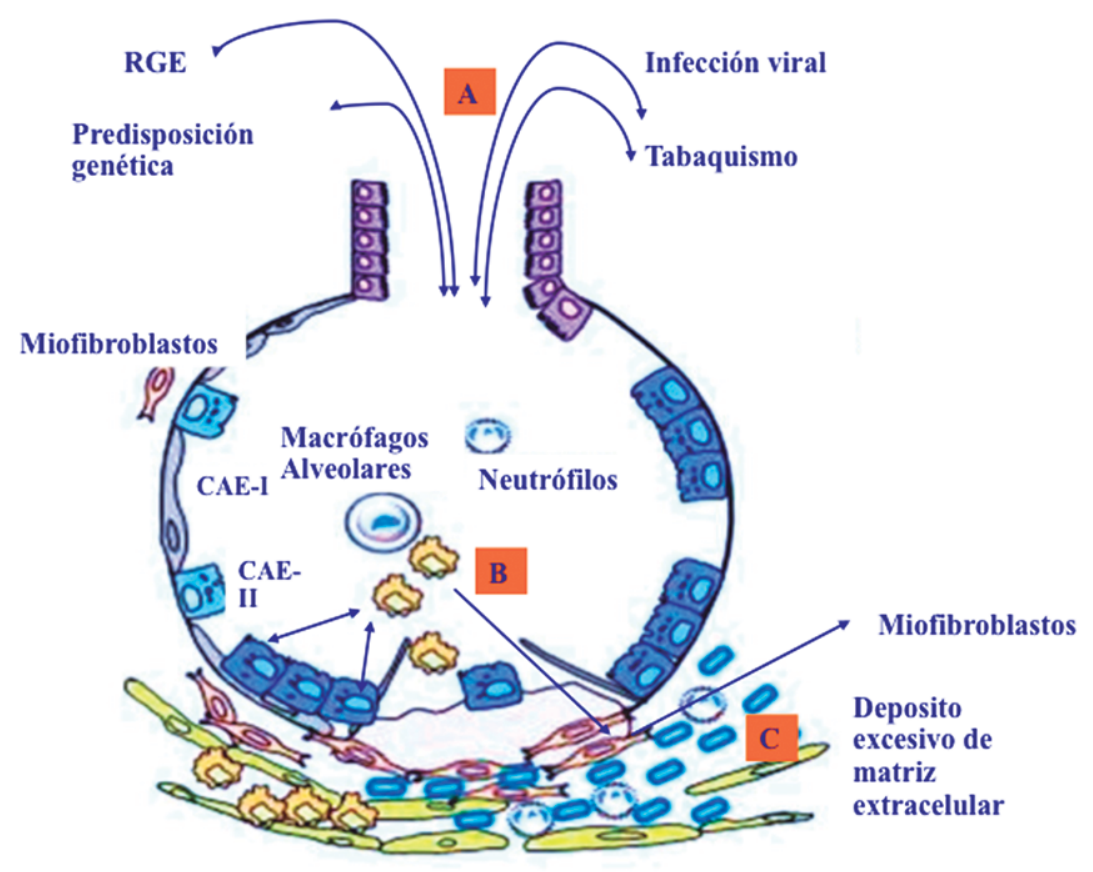

Figura 1. Patogénesis de la fibrosis pulmonar idiopátca (Adaptado de Wuyts WA et $a^{3}$ ). A. Daño: diferentes estímulos ambientales, reflujo gastro-esofágico, contaminación, tabaco, infecciones y susceptibilidad genética conducen a daño epitelial y endotelial. B. Interacción epitelio-fibroblasto: proceso de reparación caracterizado por una re-epitelización anormal, citoquinas inflamatorias, transformación epitelio-mesénquima y abundancia de miofibroblastos con formación de matriz de colágeno. C. Reparación aberrante y fibrosis: Formación excesiva de la matriz extracelular que conduce a la distorsión de la arquitectura pulmonar. RGE: Reflujo Gastro-Esofágico; CAE-I: Célula Alveolar Epitelial tipo I; CAE-II: Célula Alveolar Epitelial tipo II. 
Igualmente, la aspiración crónica secundaria a reflujo gastroesofágico (RGE) podría ser una causa (o factor contribuyente) de FPI. Sin embargo, no está claro si la alta prevalencia de RGE en pacientes con FPI está patogénicamente relacionada con el desarrollo, progresión o exacerbación de la enfermedad en el tiempo. Además, no hay certeza de que el tratamiento antiácido sea efectivo para FPI, independiente de la presencia de RGE subyacente ${ }^{11}$.

\section{Bibliografía}

1.- RAGHU G, REMY-JARDIN M, MYERS JL, RICHELDI L, RYERSON CJ, LEDERER DJ, et al. Diagnosis of idiopathic pulmonary fibrosis: an official ATS/ERS/JRS/ALAT clinical practice guideline. Am J Respir Crit Care Med 2018; 198: e44-e68.

2.- KHALIL N, XU YD, O'CONNOR R, DURONIO $\mathrm{V}$. Proliferation of pulmonary interstitial fibroblasts is mediated by transforming growth factor-beta1-induced release of extracellular fibroblast growth factor- 2 and phosphorylation of p38 MAPK and JNK. J Biol Chem 2005; 280: 43000-9.

3.- WUYTS WA, AGOSTINI C, ANTONIOU KM, BOUROS D, CHAMBERS RC, COTTIN V, et al. The pathogenesis of pulmonary fibrosis: a moving target. Eur Respir J 2013; 41: 1207-18.

4.- WOLTERS WJ, BALCKWELL TS, EICKELBERG O, LOYD JE, KAMINSKI N, JENKINS G, et al. Time for a change: is idiopathic pulmonary fibrosis still idiopathic and only fibrotic? Lancet Respir Med 2018; 6: $154-60$.

5.- WELLS AU, BROWN KK, FLAHERTY KR, KOLB M, THANNICKAL VJ; IPF Consensus Working Group. What's in a name? That which we call IPF, by any other name would act the same. Eur Respir J 2018;51:1800692.

6.- FLAHERTY K, WELLS AU, COTTIN V, DEVARAJ A, WALSH SLF, INOUE Y, et al. Nintedanib in Progressive Fibrosing Interstitial Lung Diseases. N Eng J Med 2019; 381: 1718-27.

7.- BAUMGARTNER KB, SAMET JM, STIDLEY CA, COLBY TV, WALDRON JA. Cigarette smoking: a risk factor for idiopathic pulmonary fibrosis. Am J Respir Crit Care Med 1997; 155: 242-8.

8.- HUBBARD R, LEWIS S, RICHARDS K, JOHNSTON I, BRITTON J. Occupational exposure to metal or wood dust and aetiology of cryptogenic fibrosing alveolitis. Lancet 1996; 347: 284-9.

9.- MANNINO DM, ETZEL RA, PARRISH RG. Pulmonary fibrosis deaths in the United States, 1979-1991. An analysis of multiple-cause mortality data. Am J Respir Crit Care Med 1996; 153: 1548-52.

10.- TASKAR VS, COULTAS DB. Is idiopathic pulmonary fibrosis an environmental disease? Proc Am Thorac Soc 2006; 3: 293-8.

11.- JOHANNSON KA, STRÂMBU I, RAVAGLIA C, GRUTTERS JC, VALENZUELA C, MOGULKOC $\mathrm{N}$, et al. Antacid therapy in idiopathic pulmonary fibrosis: more questions than answers? Lancet Respir Med 2017; 5: 591-8.

Correspondencia a:

Dr. Felipe Reyes C.

Email: freyes.cartes@gmail.com 